\title{
"Multifractal analysis of volatility for detection of herding and bubble: evidence from CNX Nifty HFT"
}

\begin{tabular}{|c|c|}
\hline AUTHORS & $\begin{array}{l}\text { Bikramaditya Ghosh (D https://orcid.org/0000-0003-0686-7046 } \\
\text { R http://www.researcherid.com/rid/W-3573-2019 } \\
\text { Emira Kozarević (D https://orcid.org/0000-0002-5665-640X }\end{array}$ \\
\hline ARTICLE INFO & $\begin{array}{l}\text { Bikramaditya Ghosh and Emira Kozarević (2019). Multifractal analysis of volatility } \\
\text { for detection of herding and bubble: evidence from CNX Nifty HFT. Investment } \\
\text { Management and Financial Innovations, 16(3), 182-193. } \\
\text { doi:10.21511/imfi.16(3).2019.17 }\end{array}$ \\
\hline DOI & http://dx.doi.org/10.21511/imfi.16(3).2019.17 \\
\hline RELEASED ON & Friday, 06 September 2019 \\
\hline RECEIVED ON & Saturday, 15 June 2019 \\
\hline ACCEPTED ON & Friday, 30 August 2019 \\
\hline & $(\mathrm{cc}) \mathrm{EY}$ \\
\hline LICENSE & $\begin{array}{l}\text { This work is licensed under a Creative Commons Attribution } 4.0 \text { International } \\
\text { License }\end{array}$ \\
\hline JOURNAL & "Investment Management and Financial Innovations" \\
\hline ISSN PRINT & $1810-4967$ \\
\hline ISSN ONLINE & $1812-9358$ \\
\hline PUBLISHER & LLC "Consulting Publishing Company "Business Perspectives" \\
\hline FOUNDER & LLC "Consulting Publishing Company "Business Perspectives" \\
\hline
\end{tabular}

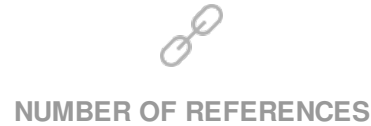

19

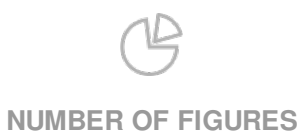

10
=:-:

NUMBER OF TABLES

3

(C) The author(s) 2021. This publication is an open access article. 


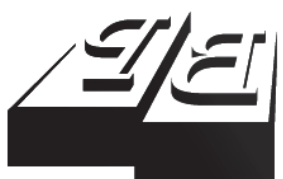

BUSINESS PERSPECTIVES

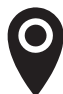

LLC "CPC "Business Perspectives" Hryhorii Skovoroda lane, 10, Sumy, 40022, Ukraine

www.businessperspectives.org

Received on: $15^{\text {th }}$ of June, 2019 Accepted on: $30^{\text {th }}$ of August, 2019

(C) Bikramaditya Ghosh, Emira Kozarević, 2019

Bikramaditya Ghosh, Ph.D., Associate Professor, Department of Finance, Christ Institute of Management, Christ University, India.

Emira Kozarević, Ph.D., Professor, Department of Finance and Financial Policy, University of Tuzla, Bosnia and Herzegovina.
MULTIFRACTAL ANALYSIS

\section{OF VOLATILITY FOR DETECTION OF HERDING AND BUBBLE: EVIDENCE FROM CNX NIFTY HFT}

\begin{abstract}
This study delves into the herding and bubble detection in the volatility domain of a capital market underlying. Furthermore, it focuses on creating heuristics, so that common investors find it relatively easy to understand the state of the market volatility. Hence, it can be termed that this study is focused on the specific financial innovation regarding bubble and herding detection coupled with investor awareness. The traces of possible volatility bubble emerge when it is positioned against its own lags (both lag1 and lag2). The volatility trigger indicated clear traces of herding and an embedded parabola function. Continuous and repetitive parabola function hinted at a subtle presence of "fractals". Firstly, the detrended fluctuation analysis has been used with its multifractal variant. Secondly, the regularized form of Hurst calculation and analysis have been used. Both tests reveal the traces of nascent bubble formation owing to prominent herding in CNX Nifty HFT environment. They also indicate a clear link with Hausdorff topological patterns. These patterns would help to create heuristics, enabling investors to be aware of possible bubble and herd situations.
\end{abstract}

Keywords

econophysics, multifractal detrended fluctuation analysis, generalized Hurst exponent

JEL Classification B16, B23, C52

\section{INTRODUCTION}

It has been noticed quite often that one domain finds its answer in the realm of entirely different domain with little or no direct connection. Volatility bubble and therefore herding behavior underneath such bubble are the genesis of an unstable capital market from time to time. The stability of the capital markets is essential for investor safety and security. Therefore, it becomes essential to create mathematics-based heuristics as a tool of financial innovation, so that the stability conditions are clearly spelt out. Classical financial models based on Gaussian distribution would find it rather difficult to explain precipitous events and the turbulence caused therefore. Multifractal patterns aren't just useful for finding coastlines of an isolated island, but also in the price changes of stocks. They may not be able to predict future with $100 \%$ accuracy, but at least could represent a realistic look of various risks in the market.

This humble attempt starts with two novel ideas. Namely the multifractal and the volatility proxy for capital markets. The concept of the "financial Reynolds number (ReHFT)" as an econophysical proxy for stock market volatility has been established through specific study. The idea germinated from a bud nestled inside fluid mechanics for more than a century. Similar analogy was found and reconstructed 
from the cradle of physics to the helm of quantitative financial domain. It generated the proxy for stock market volatility (read as "the financial Reynolds number") through an econophysical investigation. ReHFT was found to be range bound mostly for the CNX Nifty except infrequent large explosions. Moreover, those explosions were also found to have a power law connection. Even the traces of herd mentality were witnessed in a monofractal investigation. However, monofractal have their own limitations, which is why the need for this completely new quest to identify herding traces and plausible nascent bubble formation in CNX Nifty high frequency domain (ReHFT) came to the fore. "Fractals" are a tiny representation of a system mostly found to be coupled together with the concept of "scaling". They have been used from the calculation of the British coastline to the possible cotton price bubble.

\section{LITERATURE REVIEW}

Fractal theory was introduced by Mandelbrot in 1977 (Mandelbrot, 1977), although he coined the very term sometime in mid-1960s (Mandelbrot, 1963). The term "fractals" comes from the Latin word "fractus", meaning broken shreds of an object. Fractals are geometrical, complex bodies, with a unique feature of scaling inbuilt in them. They can be used to figure out the coastline of Britain, for any linguistic clarity and more interestingly to identify the financial crashes. The unique dimension of fractals could be defined in two diametrically different methods. The first one being perfectly suitable to regular fractals as 'similarity dimension', and the second one appears more general in nature, since it's an adaptation from the 'Hausdorff-Besicovitch dimension', which is applicable to not so regular fractals (Mandelbrot, 1977; Stefański, 1985). In 1997, Mandelbrot along with two other scientists have (Mandelbrot et al., 1997) created an uniquely faceted 'multifractal model of asset returns' or MMAR in order to explain and deliberate the queer variation of most financial asset closing prices (Safari et al., 2009). They furthered by pointing out that continuous multifractal analysis could well be generated for highly volatile and almost improbable transactions. This could surely resurface much information about the predicted values and market trends. 'MMAR' was projected as an alternative method to famed ARCH (autoregressive conditional heteroscedasticity) family of models to prove many stylized facts (i.e. the statistical traits of financial time series (Jiang et al., 2018), such as returns, volatility, trading volumes, recurrences intervals, etc.) as possible, such as fat tails, volatility clustering, long-term memory characteristics, self-similarity, etc. (Mandelbrot et al., 1997). It estimates the variation of daily returns in a truly complex process involving varied versions of fractal Brownian motion (Safari et al., 2009). The model has the presence of long memory and the traits of fractional Brownian motion (FBM). Moreover, this model exhibits long memory in the absolute value of price variations.

Mandelbrot's proposition of extending fractals from measuring coastlines to elucidating the movement of stocks germinated this piece of work (Mandelbrot, 1999). He argued that these techniques may not be able to predict the stock price with $100 \%$ accuracy for the following day, however, they provide estimates of precise probability so that investors can be well aware before any 'Black Swan' events. These kinds of financial innovation become essential in a brittle world of nano-second interconnectivity. Too much information flow too quickly is making the financial system unstable. Such innovations pave the way for predicting and restoring stability.

One eminent statistician from Rice University (Riedi, 1999) had published his technical report "Introduction to Multifractals", in which he stated that multifractals analysis is focused on describing local unitary movement of measures or specific functions in a queer geometrical way. A specific method of multifractal analysis, multifractal detrended fluctuation analysis (MFDFA) is proposed by an eminent scientist (Kantelhardt, 2008), and it could illustrate various embedded statistical traits of a stochastic series on varied time scales. MFDFA is one of the most efficient ways to scrutinise whether an apparent non-stationary series is cardinally multifractal in nature or not. It usually considers changing 'average volatility' of any stochastic series through all of its intervals. Moreover, it generates the unique volatility function and thus investigates the generalized Hurst exponents (Hurst, 1951) based on the power law driven volatility attributes. 
Inspired by its compelling metaphor with fluid turmoil, multifractal detrended analysis of financial markets blossomed, composing one of the fundamental pillars of econophysics (Jiang et al., 2018). Spanish research group consisting of the likes of Suárez-García and Gómez-Ullate's maiden endeavor of testing the multifractality exhibited by the famed high-frequency returns of IBEX35 (Suárez-Garcíaa \& Gómez-Ullate, 2014), spanning over a crucial two-year period starting from 2009 till 2010. As far as MFDFA is concerned, it has been observed that the scaling exponents and its individual spectrum both indicated that the underlying stochastic time series exhibits a clear trace of multifractality. It's a known fact that wide 'singularity spectrum' often indicates presence of long memory in the time series. High frequency of arrival of information in a given state of market often causes 'singularity spectrum' depicting and establishing a clear 'long memory'. Information reaches the market on a daily basis. These information gets superimposed at a higher frequency. Further that generates bubble and herding, which is evident from the multifractality test. If information gaps are far too long and they seem to be having relatively less impact then the multifractality test doesn't show a clear 'singularity spectrum'. A group of Chinese researchers (Wang et al., 2014) have found that due to the complex nature of the markets and their increasing high connectivity in today's well-connected world, it's quite difficult to find a clear pattern despite the so called 'efficient market theory. Since, the assumptions sometimes seem way too theoretical. Thus, the call of the day is to find a clear pattern for prediction and control connected networks of global markets. Even stability calculation becomes way too important as well. The same Chinese group of researchers analyzed daily returns of NASDAQ Composite Index. They've considered a time period that starts immediately after the world credit fiasco. Soon they came to a conclusion that in no way the returns fit Gaussian distribution. In fact the fat tails were quite evident in this case making the prediction way too difficult. They found profound trace of multifractality. Furthermore, they found that the long memory trait is the cardinal reason behind the embedded multifractality in NASDAQ. They furthered by confirming that the entire journey of NASDAQ returns may not be entirely stochastic, but it's purely stochastic in certain periods. Some eminent American studies such as Los and Lipka (2003) found that most European stock markets follow anti-persistent way (indicating entirely stochastic and almost no traces of herd behavior) while empirically testing the degree of persistence of daily index returns of eight European bourses.

This paper is based upon econophysical volatility proxy rather than daily returns or log normal returns of indices. MFDFA has been used to study the scaling behavior of non-stationary multifractal time series in this case. Non-stationary time series often suffer from trend due to the shift of the dynamic mean value. Thus, detrending is required to understand the absolute movement and identification of any cycle (whether hidden or not). The main idea underlying this procedure consists of taking into account deviations of $q^{\text {th }}$-order moments from polynomial fits. In fact, notice that for $q=2$, classical DFA remains as a particular case of MFDFA. Recollection factor over different orders shows the consistency of the long memory process, i.e. "mostly it has been observed that the autocorrelation function or ACF generated from the absolute returns of any stochastic series decompose quite slowly; while being the function of the time lag, following a specific power law" (Safari et al., 2009), as well. One latest study in the said context shows evidences of short-long dependence and light-heavy tail (fat tails) fluctuations for financial time series (especially for certain specific stocks in the US). Moreover, they've verified the accuracy across various version of Weiner Process (modified Brownian motion) and more appropriate fractional Brownian motion to find that MFDFA results to be consistent enough to rely upon (Thompson \& Wilson, 2016).

\section{METHODOLOGY}

Taking previous research into consideration, two embedded assumptions paved the way for this entire work. Though monofractal were put into use effectively and predictability (in form of profound herding) was confirmed yet, it was never full proof. Monofractal precisely depict an incomplete narrative. Moreover, monofractal fail to track for any time series that has the underlying of asset prices due to multiple dimensions 
and their ever-changing nature. Hence, ReHFT cannot be tracked using monofractal alone. Secondly, these stochastic series are self-similar in some way, thus ignoring a Markov chain, random walk, and generalized Brownian motion (GBM) basic assumptions. Though technically ReHFT is not a time series based on stock market closing, all its ingredients are traced back to the CNX Nifty HFT domain. Certain trailblazing literature found links of power law relationship of asset prices and their far-fetched implications to stochastic nature along with the embedded persistent pattern (indicating presence of herd). Mandelbrot (Mandelbrot et al., 1997) found that patterns in the price follow power law relationship and asset prices were far from normal. He also found that the Lévy stable condition or $\alpha$ range from 0 to 2 , where $\alpha=2$ satisfies the condition for embedded Gaussian distribution. Mandelbrot further developed another path-breaking invention when he constructed "Rescaled Range Analysis" $(R / S)$. The Hurst exponent follows $H=1 / \alpha$; thus, when $\alpha=2$, the Hurst exponent becomes completely stochastic or in other words follows Brownian motion completely. In other expression, $H=0.5$ should ideally follow a Gaussian distribution curve.

\subsection{Multifractal detrended fluctuation analysis (MFDFA)}

Mandelbrot's trailblazing 'fractals' were furthered by a group of eminent researchers (Kantelhardt et al., 2002) who gave a clear shape to the entire process of identification of impact of multifractality in a noisy time series. From bio-medical series to stochastic financial series, multifractal is used everywhere. Initially pure noisy time series have to be amended enough to have an appearance of a 'random walk' type series. Consistent noises could be translated to pure 'random walk' series by subtracting the mean value first followed by integration of the same (Ihlen, 2012). Calculation for the 'RMS' or the root mean square variation of the same series holds the key in this entire process. Locally these RMS values are calculated (especially in the areas that are having clear trends) and then summarized as an entire RMS. However, this calculation is partially overshadowed with substantially large oscillations for tiny samples and vice versa. Overall RMS and the various samples will ideally show their 'power law' connection explicitly here. This is the famous "monofractal detrended fluctuation analysis" or DFA. The coefficient for this specific relation is none other than the "Hurst exponent' (Hurst, 1951; Watkins \& Franzke, 2017). This same method once extended to the $q^{\text {th }}$ order becomes 'multifractal detrended fluctuation analysis" or MFDFA (Ihlen, 2012). Fractal properties and their coefficient values do change from mono to multi, the latter being more accurate in nature.

\subsection{Generalized Hurst exponent (GHE)}

While British hydrologist was working on the River Nile project in 1951, he found the longrange correlations in a time series (Hurst, 1951). Although this trailblazing tool was born in the cradle of water storage problem, yet it found apt usage in various fields such as chaos theory, spectral analysis, fractals, and long memory processes. It confirms both correlation and persistence. This makes the Hurst exponent an excellent index for studying complex financial time series.

The robustness of such mathematical construct is largely based on the $q^{\text {th }}$-order moments for the distribution of incremental value. That incremental value has been modeled through a stochastic process. This method provides information about evolution of any financial time series. The scaling pattern leads this entire calculation to the wellknown generalized Hurst exponent (GHE). It has been further noted that all the scaling traits of series under consideration are usually embedded in $H(q)$, that can be termed as 'scaling index'. Anyways, this in turn consolidate the fundamental premise stating the confirmatory power of GHE over its peers. It has been found that such stochastic series will have a unique scaling property represented by " $H$ ", which in turn matches perfectly with the selfaffine exponent (or self-similar exponent) for that same time series. However, the quantitative measure of self-similarity (Hurst coefficient or exponent) is found to be constant for each $q^{\text {th }}$ order moment. Researchers have found GHE to be precisely closer to the actual Hurst exponent (depicting both herding and bubble) for $q=1$. Since a higher degree of "q" has been considered, thus both GHE and MFDFA output are considered. In fact, for a 
higher " $q$ " order MFDFA becomes more accurate to determine self-affinity (not only self-similarity; defined later in detail).

Zones of the Hurst exponent are defined in Table 1.

Table 1. Zone of the Hurst exponent

\begin{tabular}{l:l}
\hline Hurst exponent & \multicolumn{1}{c}{ Interpretation } \\
\hline $\mathrm{HE}<0.5$ & $\begin{array}{l}\text { Antipersistent, no shape, no herd behavior, } \\
\text { fractal quotient is lesser }\end{array}$ \\
$\mathrm{HE}=0.5$ & $\begin{array}{l}\text { Follows theoretical random walk, entirely } \\
\text { stochastic in nature }\end{array}$ \\
$\mathrm{HE}>0.5$ & $\begin{array}{l}\text { Evidently persistent, clear shape, trace of } \\
\text { herd behavior, fractal quotient is higher }\end{array}$ \\
\hline
\end{tabular}

\section{RESULTS AND DISCUSSION}

Figure 1 shows a pure theoretical white noise and can be termed as a pure stochastic array of observations forming a random walk pathway (the lower panel in Figure 1), a uni-fractal random walk pathway has been depicted with one fractal dimension (the middle panel in Figure 1), and a multi-fractal random walk pathway (the upper panel in Figure 1). Fractal properties (roughness in other words) and volatility of the embedded time series (in this case its 'financial Reynolds number' for CNX Nifty HFT domain) were calculated from February 2012 to December 2016. A zoom in image opens up the true roughness of a multifractal analysis. The rationale is pretty clear as well. The number of direct, indirect, and even latent variables responsible for the volatility (the 'financial
Reynolds number' as an econophysics proxy) is far too many to consider at any instance; hence, the roughness is found to be on the higher side.

Local detrending of the time series (volatility proxy) is depicted very clearly in Figure 2. Three distinctly different polynomial trends are visible here. They seem linear when $m=1$, looks like a quadratic when $m=2$, and transforming to cubic when $m=3$.

DFA cardinally establishes the monofractal nature of any stochastic series. It is often represented by a unique power law connection between various error calculations (read as RMS) across various scales. Furthermore, it has been observed that this unique relationship is depicted by the slope $(H)$ of the regular regression line (see Figure 3).

Figure 4 demonstrates the slope, $H$, of the regular regression line famously described as the Hurst coefficient or exponent (Hurst, 1951). It identifies the embedded monofractal nature by examining the pace of RMS, $F$ of local variations advance against the expanding sample size or, in other words, "scale". It has been depicted that RMS is advancing ahead quicker with the sample size for the time series with varied fractal dimension when in comparison with white noise time series.

The overall $q$-order RMS is able to differentiate between the micro-structure of tiny and relatively substantial oscillations. Further its able to differentiate between varied fractal time series under

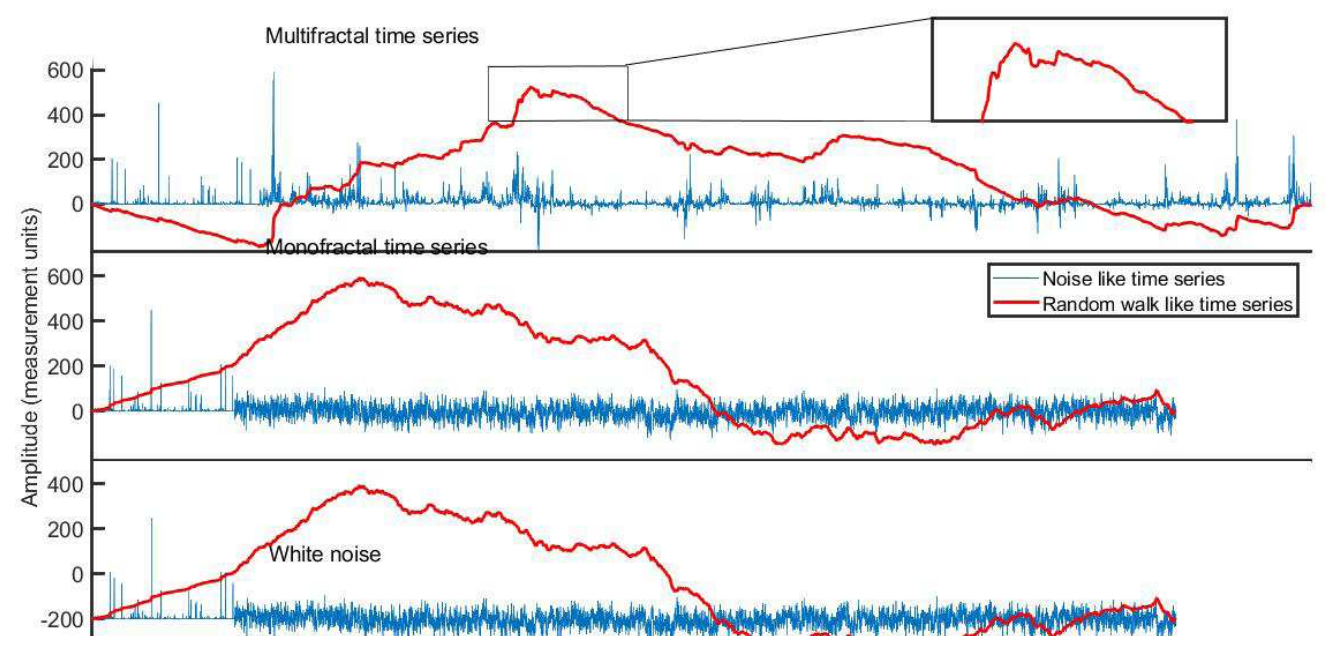

Figure 1. Multifractal, monofractal, and white noise like time series 


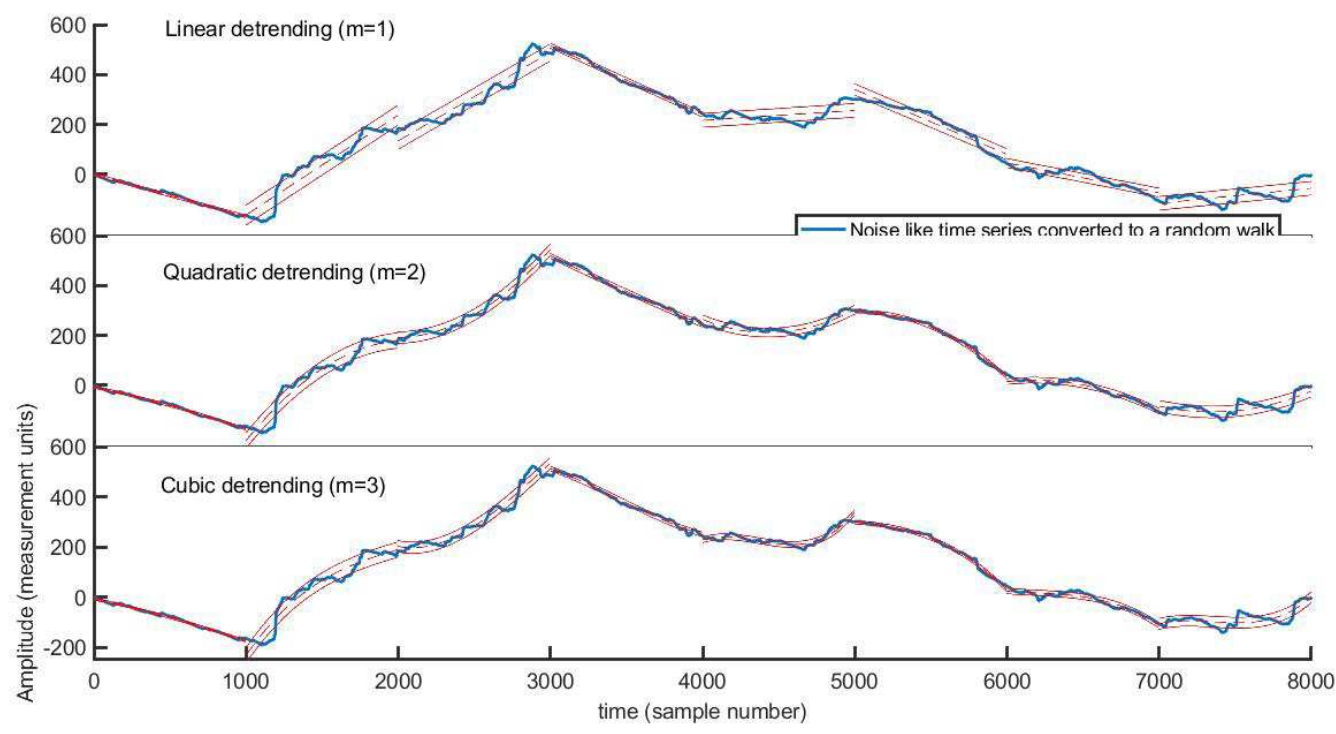

Figure 2. Cubic, quadratic, and linear detrending

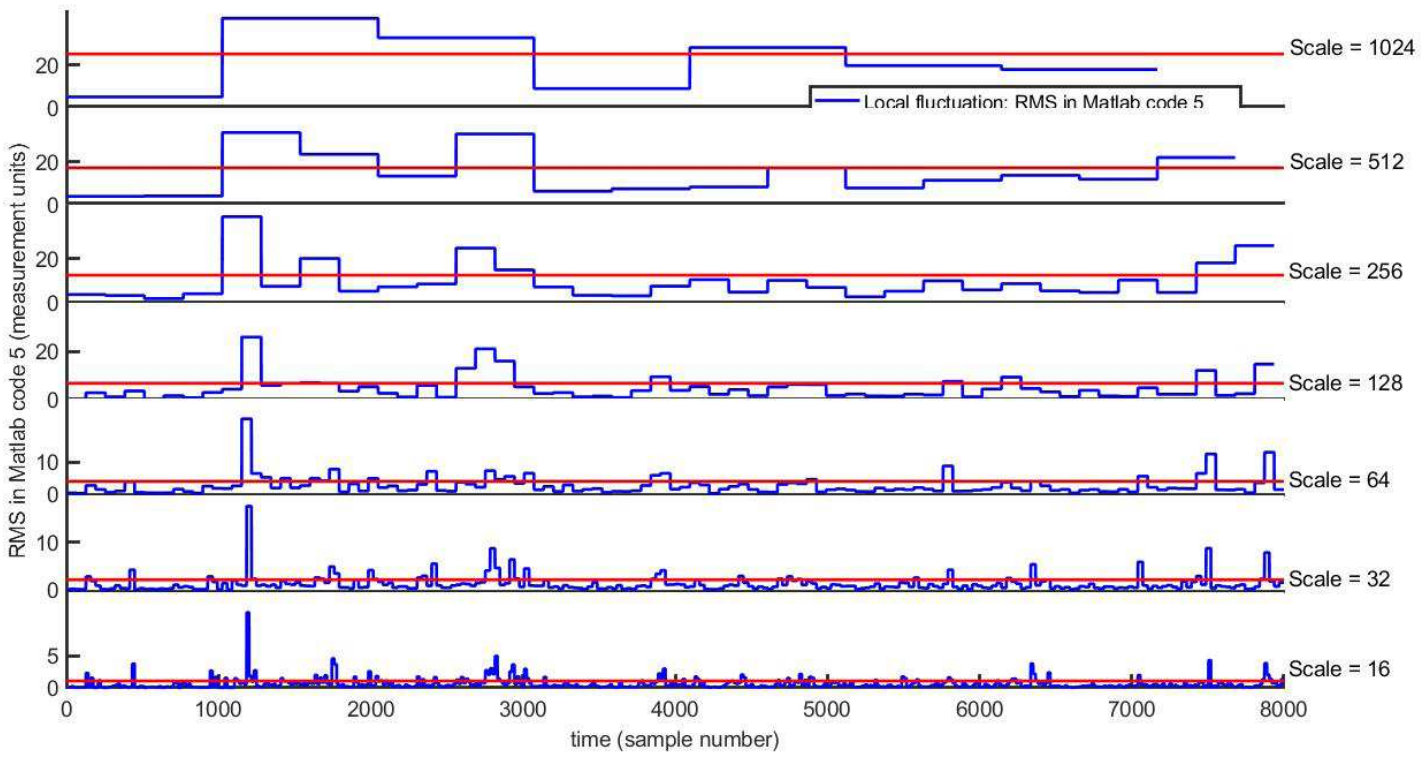

Figure 3. Root mean square (RMS)

consideration (Ihlen, 2012). The multifractal time series have been found to be having local oscillations with extremely tiny and relatively large magnitudes, which is absent in the monofractal time series. Hence, the monofractal time series is found to have normal distribution. The multifractal time series is not normally distributed and all $q^{\text {th }}$-order statistical moments should be considered. The periods with large variations are virtually blank for monofractal, but not so for multifractal analysis.

The $q^{\text {th }}$-order Hurst exponent has been expressed as ' $\mathrm{Hq}$ ', for the time-series under consideration has been found to have various different traces; the multifractal one (blue trace), monofractal one (red trace), and finally white noise (turquoise trace), (Ihlen, 2012) where the colored dots represent the slopes $\mathrm{Hq}$ for various order of the exponent such as $q=-3,-1,1$, and $3 . q^{\text {th }}$-order Hurst exponent for different time series is illustrated in Figure 6 (AC). It has been found that $H q$ (the $q^{\text {th }}$-order Hurst exponent) comes down from 1 to 0.77 , when scaling is done on the upside for various order of the exponent such as $q=1,2,3$, etc. Hence, the larger 


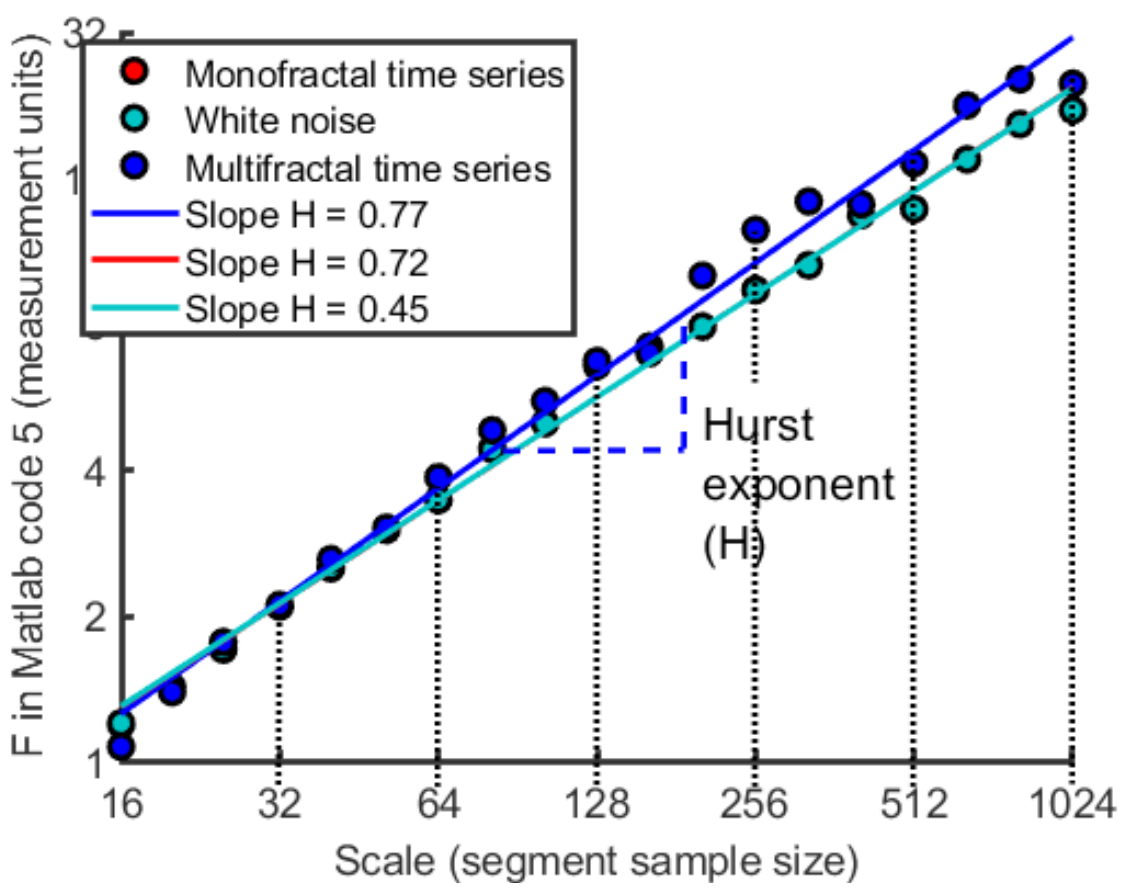

Figure 4. Hurst exponent

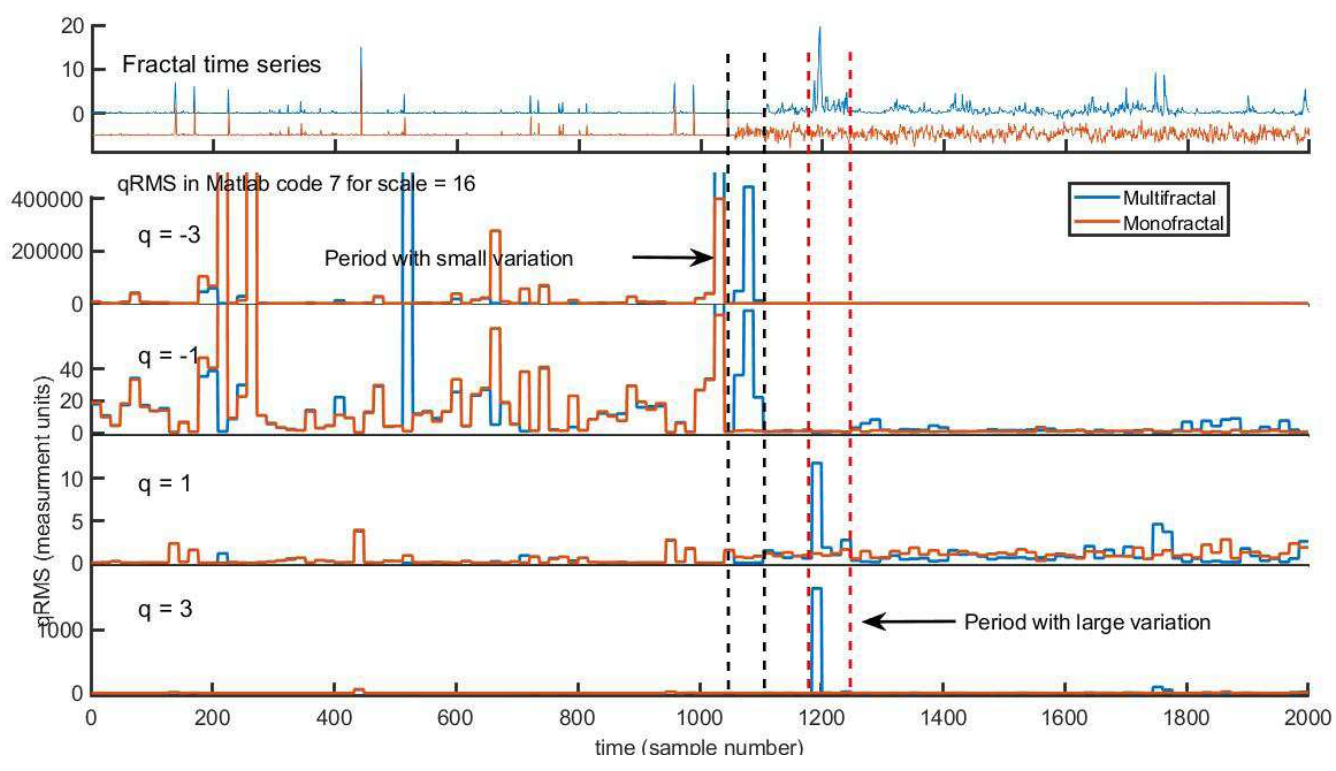

Figure 5. $q^{\text {th }}$-order RMS for different time series

the sample size, the less the persistence. However, persistence denoted by $\mathrm{Hq}$ or the Hurst exponent gets stabilized around 0.6 , which is still inside the persistence zone (see Figure 6).

The plot of $h q$ versus $D q$ is referred to as the "multifractal spectrum" (Ihlen, 2012; Vardhini et al., 2018). The multifractal spectrum is not found to be skewed (Proto, 2012). Moreover, $D q$ goes up with $h q 0$ upto $h q>=1$ (Figure 7), this confirms the assumption that stock markets are multifractal, not monofractal. This in turn indicates similarity with the embedded volatility pattern, which incidentally resembles an inverted parabola.

A multifractal spectrum could well be viewed as an ensemble of independent monofractal for the same time series. When that Hurst exponent 

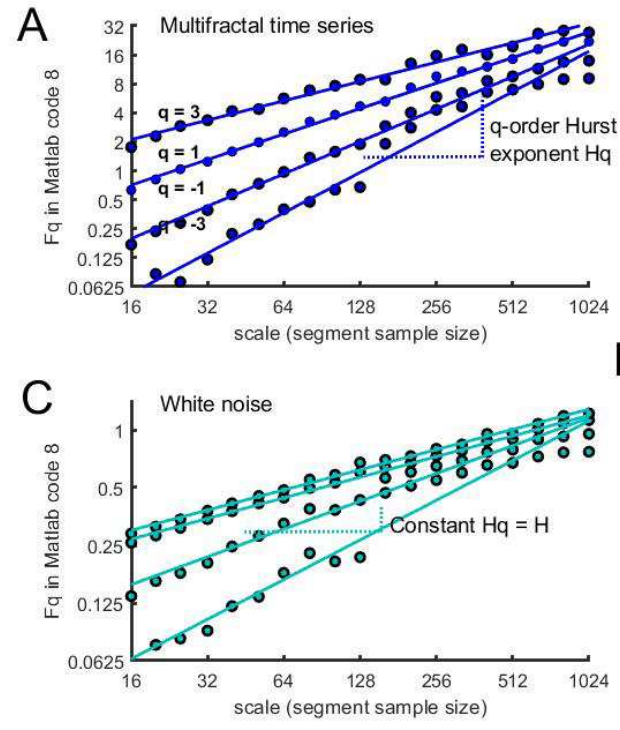
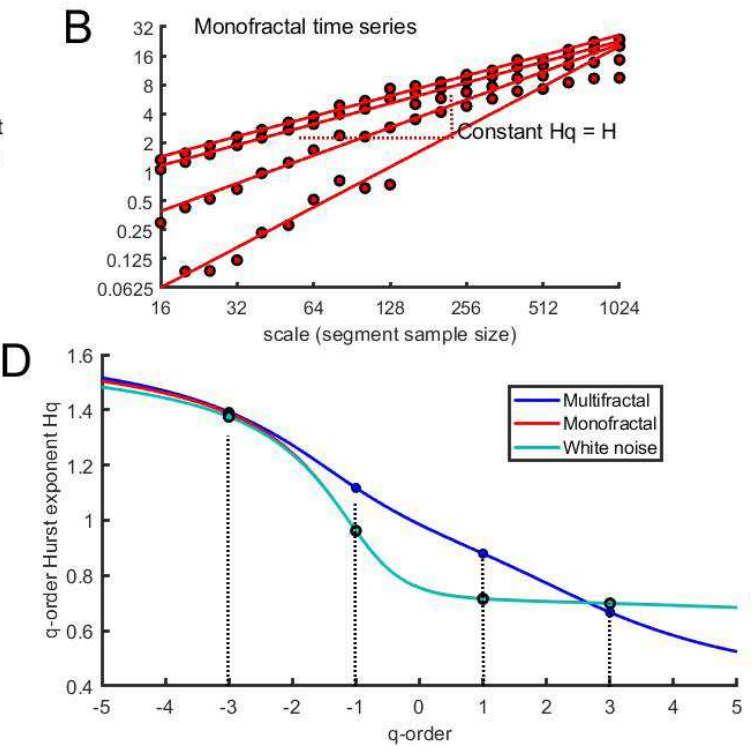

Figure 6. $q^{\text {th }}$-order Hurst exponent for different time series

stays close to 1, it confirms long memory process. ReHFT is found to have long memory, as well as it has been found to have a Hurst exponent of around 0.77 . Interestingly it has been observed that persistence is consistent, which makes volatility predictability possible in a continuous basis. The symmetry, which has been observed in the multifractal spectrum, proves the self-affine nature of ReHFT. This means that ReHFT depends on many parameters or dimensions, but it follows its own past patterns as well. However, the degree of following or the impact of dimensions does change in this long memory stochastic process. Hence, it is difficult to anticipate which dimension will play the key role in future. It is a kind of a distorted self-similar process.

Figure 8 depicts a picture of estimation of the Hurst coefficient or exponent in the sample vicinity. Both the local minimum and local maximum Hurst coefficient or exponent respectively emerge out as the slope of the respective upper and lower red lines (Ihlen, 2012). This converges from the local maximum and local minimum of RMS straight to the regular regression line.
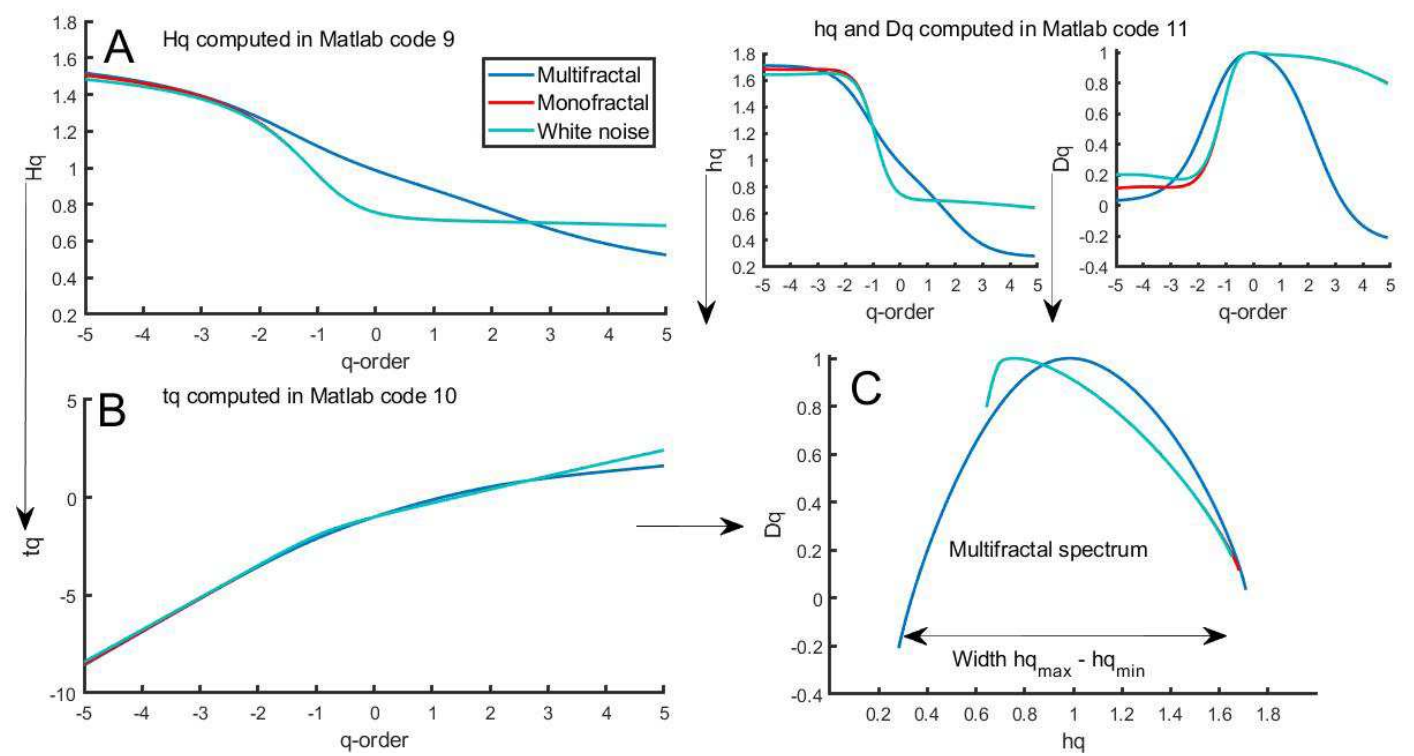

Figure 7. The multifractal spectrum of time series 

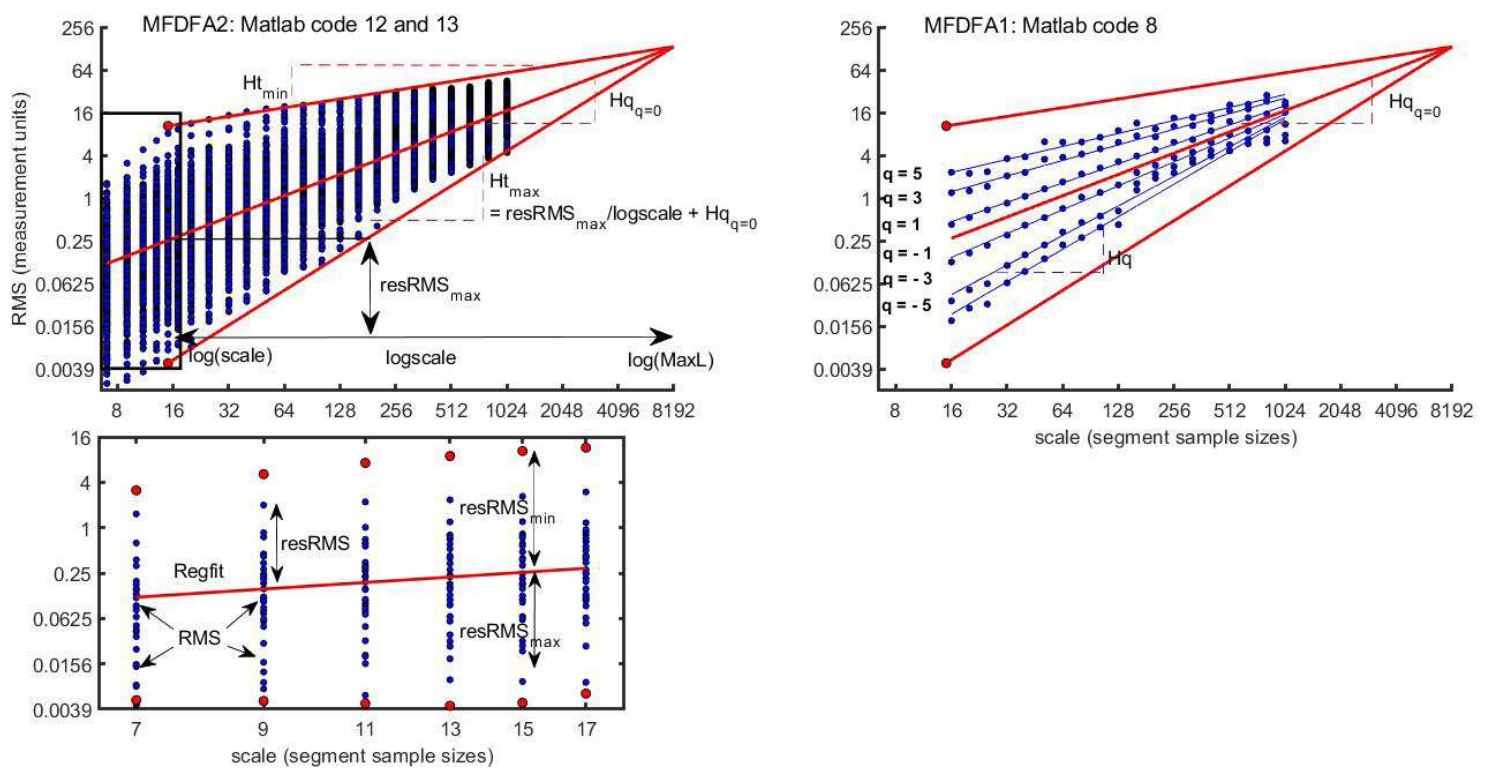

Figure 8. Estimation of the Hurst exponent

Self-similarity and self-affinity are different. A tiny sample grows symmetrically in all possible directions in case of self-similar substance or sample. However, in case of self-affinity, the growth is asymmetrical in nature; it exhibits non-uniform growth across the sample. GHE results are summarized in Table 2.

Table 2. GHE output

\begin{tabular}{c|c|c|c}
\hline $\begin{array}{c}\text { Observation } \\
\text { range }\end{array}$ & $\begin{array}{c}\text { Hurst } \\
\text { exponent }\end{array}$ & $\begin{array}{c}\text { Fractal } \\
\text { dimension }\end{array}$ & $\begin{array}{c}\text { Change } \\
\text { in volatility }\end{array}$ \\
\hline $1-105$ & 0.59961 & 1.40039 & $-39 \%$ \\
\hline $106-305$ & 0.88042 & 1.11958 & $1.27 \%$ \\
\hline $306-505$ & 0.64146 & 1.35854 & $-66 \%$ \\
\hline $506-705$ & 0.63717 & 1.36283 & $130 \%$ \\
\hline $706-905$ & 0.61342 & 1.38658 & $215 \%$ \\
\hline $906-1056$ & 0.81664 & 1.18336 & $-81 \%$ \\
\hline
\end{tabular}

The average Hurst exponent is 0.69812 and the correlation between the changes in volatility with the Hurst exponent is 0.657 .

Whenever the change in volatility is found to be higher, it coincides with the zones where the Hurst exponent is relatively lower (see Table 1). It is plausible as lower Hurst indicate lower herd behavior. Therefore, the secular direction will not be possible. Hence, volatility will change at a higher pace. Volatility means random track changes in a time series with rapid frequency and inconsistent amplitude. Higher Hurst exponent indicates secular movement with less frequency, less change of track, and consistently higher amplitude (virtually indicating a clear pattern).
Another interesting fractal discovery was explained during the course of this analysis. Fractal dimensions are linked with Hausdorff topology connection. This in turn is going develop the 'investor heuristics', essential for the understanding about the current market condition.

Table 3. Fractal geometry and topology connection

\begin{tabular}{c|l}
\hline $\begin{array}{c}\text { Fractal } \\
\text { dimension }\end{array}$ & \multicolumn{1}{c}{$\begin{array}{c}\text { Fractal geometry similarities } \\
\text { (in shapes and patterns) }\end{array}$} \\
\hline 1.40 & $\begin{array}{l}\text { Douady rabbit, as per Hausdorff } \\
\text { Douady rabbit, as per Hausdorff }\end{array}$ \\
\hdashline 1.38 & $\begin{array}{l}\text { Five circles inversion fractal, as per } \\
\text { Hausdorff }\end{array}$ \\
\hline 1.36 & $\begin{array}{l}\text { Five circles inversion fractal, as per } \\
\text { Hausdorff }\end{array}$ \\
\hline 1.36 & $\begin{array}{l}\text { Dendrite Julia set, as per Hausdorff } \\
\text { Contour of the Gosper Island, as per } \\
\text { Hausdorff }\end{array}$ \\
\hline 1.18 & \\
\hline 1.12 &
\end{tabular}

Interesting observations surface out following Hausdorff topology methods and linking them with Julia sets and Mandelbrot fractals (Hausdorff, 1919). Hence, as herding increases, the shape changes from a Douady rabbit (see Figure 9) to a dendrite Julia set (see Figure 10). Thus bubble formation possibilities could be at the anvil when Douady rabbit formation slowly takes the shapes of Julia sets and is closely followed by the contour of the Gosper Island. On the contrary, predictability (persistent patterns) would surely take a nosedive in these cases. 


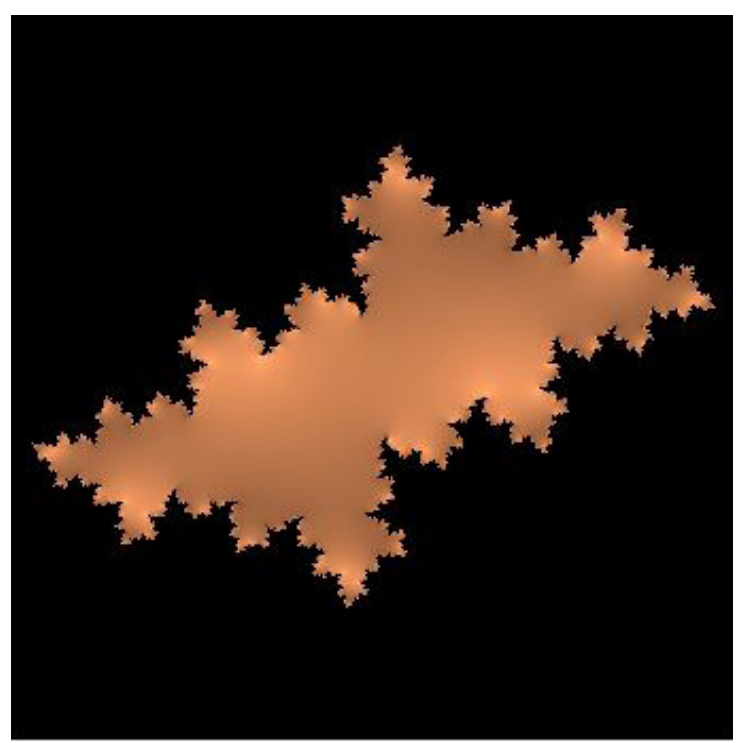

Figure 9. Douady rabbit

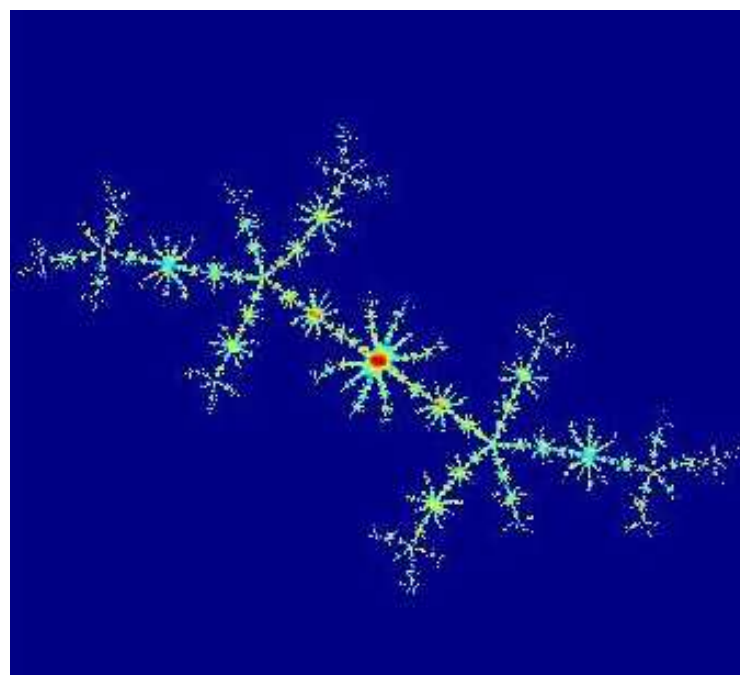

Figure 10. Dendrite Julia set

Douady rabbit, having a fractal dimension of around 1.4, ensures lower degree of persistency of Hurst exponent. This in a way indicates that volatility is persistent with a lower degree. Hence herding possibilities are found to be less. Volatility driven bubble possibilities are found to be less as well. This financial innovation would surely give a clear-cut idea about the capital market condition to an average investor.

The dendrite Julia set confirm implicit persistent pattern along with profound herd behavior and possible nascent volatility bubble. Average inves- tors can be aware of such a diagram since it signifies instability and excess volatility for them to venture.

Log returns from stock market were proven to be fractal long time ago (Redelico, 2012). It has been proved earlier that the financial crisis developed out of prominent bubble; more interestingly empirical evidence of multifractal trace (or signature) has been quite profound (Redelico, 2012). Hence, multifractal traces and prominent bubbles are cobbled together more often than not, leading to a possible catastrophe at the anvil. 


\section{CONCLUSION}

This paper proves that even the volatility follows the same trail. Through the empirical research on the 'financial Reynolds number (ReHFT)' derived out of CNX Nifty HFT domain using MFDFA, it has been revealed that ReHFT possesses strong traces of herding. The reason for this is yet to be explained. In fact, the relationship became stronger with multifractal calculation when compared to its monofractal counterpart (the Hurst exponent became 0.77 from 0.72). Multifractals (read MFDFA) were found superior for obvious reasons over their peer (i.e. GHE), however both indicated similar outcome.

The embedded fractal structure also provides a lot of information and extends evidence of an inherent fractal nature inside the econophysical volatility proxy, which has so far remained undetected. Probability of a nascent bubble formation increases with unexpectedly rapid progress under a strong influence of secular movement (indicated by the Hurst exponent). A higher level of the Hurst exponent confirms persistent patterns within the realm of quantitative finance. The embedded herd behavior inside the econophysical volatility proxy, leading to possible bubble situation has been confirmed in this paper. This work has the genesis of developing heuristics for market volatility; profound topological heuristics pattern surfaces out during this study. The dendrite Julia set, or contour of Gosper Island share the same fractal dimension as that of a possible herding and nascent bubble formation. An apparent topography indicating possible financial catastrophe remained a significant outcome. A regular retail investor too would get enough clue about the status of 'bubble' and 'herd', which is apparently hidden in the bourses. Market stability could get a new definition hence on. Log returns have been tested far often in the past, thus this brings a new perspective from the volatility standpoint. What that means is that fractals remained universal and their footprints were found everywhere from hydrodynamic fluid mechanics to bourse volatility.

\section{FUTURE RESEARCH POSSIBILITIES}

Intrinsic trends are often hidden in both stationary and even non-stationary time series. It is both difficult to identify and decipher. Most researchers have used 'feasible trend functions' to identify the same. Fractional Brownian motion and Platt Scaling could well be used further to elaborate the study, where 'volatility bubble' could be spotted. Novel approaches such as OMW-EEMD-MFDFA could well be applied on volatility proxy for advanced analysis. OMW stands for overlap moving window algorithm, which will segregate the time series in various moving OMWs.

\section{REFERENCES}

1. Hausdorff, F. (1918). Dimension und ausseres Mass. Mathematische Annalen, 79(1-2), 157-179. https:// doi.org/doi:10.1007/BF01457179

2. Hurst, H. (1951). Long-term storage capacity of reservoirs. Transactions of the American Society of Civil Engineers, 116(1), 770-799. Retrieved from https:// cedb.asce.org/CEDBsearch/record. jsp?dockey $=0292165$

3. Ihlen, E. A. F. (2012). Introduction to multifractal detrended fluctuation analysis in Matlab. Frontiers in
Physiology, 141(3). https://dx.doi. org/10.3389\%2Ffphys.2012.00141

4. Jiang, Z.-Q., Xie, W.-J., Zhou, W.-X., \& Sornette, D. (2018). Multifractal analysis of financial markets. Retrieved from http:// arxiv.org/abs/1805.04750

5. Kantelhardt, J. W. (2008). Fractal and Multifractal Time Series (59 p.). Retrieved from http://arxiv. org/abs/0804.0747

6. Kantelhardt, J. W., Zschiegner, S. A., Koschielny-Bunde, E., Havlin, S., Bunde, A., \& Stanley,
H. E. (2002). Multi-fractal detrended fluctuation analysis of nonstationary time series. Physica A: Statistical Mechanics and its Applications, 316(1-4), 87-114. https://doi.org/10.1016/ S0378-4371(02)01383-3

7. Los, C. A., \& Lipka, J. M. (2003). Long-Term Dependence Characteristics of European Stock Indices. SSRN Electronic Journal, 1-40. https://doi. org/10.2139/ssrn.388020

8. Mandelbrot, B. (1999). A Multifractal Walk down Wall Street. 
Scientific American, 280(2), 70-73. https://doi.org/10.1038/scientificamerican0299-70

9. Mandelbrot, B. B. (1963). The Variation of Certain Speculative Prices. The Journal of Business, 36(4), 394-419. https://doi. org/10.1086/294632

10. Mandelbrot, B. B. (1977). Fractals: form, chance and dimension (International Business Machines, Thomas J. Watson Research Center) (XVI). San Francisco: W.H. Freeman and Co.

11. Mandelbrot, B. B., Fisher, A. J., \& Calvet, L. (1997). A Multifractal Model of Asset Returns (Working Papers - Yale School of Management's Economics Research Network, 1). Retrieved from https://papers.ssrn.com/sol3/ papers.cfm?abstract_id $=78588$

12. Redelico, F. O., \& Proto, A. N. (2012). Empirical fractal geometry analysis of some speculative financial bubbles. Physica A Statistical Mechanics and its Applications, 391(21), 5132-5138. https://doi.org/10.1016/j.physa.2012.01.045

13. Riedi, R. H. (1999). Introduction to Multifractals. Houston. Retrieved from http://www.stat.rice. edu/ riedi/Publ/PDF/intro.pdf

14. Safari, A., \& Seese, D. (2009). Non-parametric estimation of a multiscale CHARN model using SVR. Quantitative Finance, 9(1), 105-121. https://doi. org/10.1080/14697680802039865

15. Suárez-Garcíaa, P., \& GómezUllate, D. (2014). Multifractality and long memory of a financial index. Physica A: Statistical Mechanics and its Applications, 394, 226-234. https://doi.org/10.1016/j. physa.2013.09.038

16. Thompson, J. R., \& Wilson, J. R (2016). Multifractal detrended fluctuation analysis: Practical applications to financial time series. Mathematics and
Computers in Simulation, 126, 63-88. https://doi.org/10.1016/j. matcom.2016.03.003

17. Vardhini, P., Punitha, N., Navaneethakrishna, M., \& Ramakrishnan, S. (2018). Multifractal Analysis of Term and Preterm Uterine EMG Signals Using Wavelet Leaders. In IEEE Life Sciences Conference (LSC) (pp. 271-274). http://dx.doi. org/10.1109/LSC.2018.8572107

18. Wang, W., Liu, K., \& Qin, Z. (2014). Multifractal Analysis on the Return Series of Stock Markets Using MF-DFA Method. In 15th International Conference on Informatics and Semiotics in Organisations (ICISO, 2014) (pp. 107-115). Shanghai: Springer.

19. Watkins, N. W., \& Franzke, C. (2017, August). A brief history of long memory: Hurst, Mandelbrot and the road to Road to ARFIMA, 1951-1980. Entropy, 19(9), 437. https://doi.org/10.3390/e19090437 